\title{
Design and Construction of the Virtual Cloud Platform for the Laboratory
}

\author{
Wang Kefeng ${ }^{1}$ and Ye FeiYue ${ }^{2}$ \\ ${ }^{12}$ (School of Computer Engineering, Jiangsu University of Technology, \\ Changzhou 213001, China) \\ E-mail:1'wkf88@163.com, ${ }^{2} 11404105 @ q q . c o m$
}

\begin{abstract}
Construct a virtual cloud platform laboratory, based on the cloud technology. Introduce the design, construction and architecture of the cloud platform in detail. Analyze the advantage and security of the cloud platform. It has low cost of hardware and software, small late maintenance, and centralized data management, the security of data is greatly improved, compared with the traditional computer $(P C)$ laboratory. Meanwhile the virtual platform also has the all characteristics of the real computer. The virtual cloud platform of the laboratory is the trend and direction of the development of the computer lab in the future.
\end{abstract}

Key words: virtual; cloud; computer; platform; design of laboratory

\section{Introduction}

The construction of enterprise network, almost all adopts the PC mode. More and more problems appeared in the process of building the PC network such as the late maintenance workload, high cost of software and hardware upgrade, data security, distributed management and so on, and the large number of important or confidential data distribution in each PC, is not conducive to data centralized management .

Desktop virtualization is the rapidly emerging trend as virtualization technology; more and more enterprises begin to accept the desktop virtualization technology to realize the enterprise desktop infrastructure. It is made out several virtual machine (VM) based on the desktop virtualization technology on a powerful server, the user or administrator can install the operating systems, applications, and so on in the virtual machine, one or more virtual machine can be assigned to one or more users by the administrator based on the need, while users no longer need to deploy powerful PC, and as long as cloud computing terminal is safe and easy to manage and can be connected to the virtual machine in the data center and use the virtual machine, through the network the virtual host on the server can be transferred to the client and to the end user [1].

The "cloud terminal + cloud desktop", is provided for users with "one-stop" work style of desktop virtualization solutions, greatly simplifies the deployment and management of desktop. Reduce the cost of desktop management by saving operating expenses and improving flexibility, at the same time provides the ultimate user experience of the Pc, enterprise level desktop reliability, data protection and disaster recovery ability

\section{The Overall Architecture Designing of Desktop Cloud Platform}

The whole desktop cloud solution is composed of the cloud terminal thinclient, virtual desktop controller OVD, virtual machine OVP, servers, storage equipment and so on, is realized the enterprise staff's office desktop unified deployment on the server, the employee's personal data is stored centrally, and then through the network (LAN or wan) desktop system will be fast delivered to employees, employees can access to the office by 
a variety of different types of terminal equipment such as thin client, laptop, mobile phone and tablet at anytime and anywhere, thus build a new desktop office mode.

CDesktop OVD desktop cloud platform is delivered to any user as an on-demand service in the form of the exclusive desktop at any time and at any place, the desktop, using unique VDX desktop transport protocol, the entire desktop can be quickly and safely delivered to the all users inside the business enterprise by OVD, no matter they are fixed office staff, or mobile office staff, specific platform structure is shown in figure 1. Platform core components are as follows:

Virtual machine management platform OVP is built as server cluster environment that hardware resource can be dynamic scheduling, through the virtual machine can install Windows and Linux desktop operating system and application, realize the unified management and performance monitoring of desktop pool.

Unique symmetric cluster architecture can be logged on to any one server to manage the whole cluster without installing separate central management server, thus ensuring the high availability of the platform.

OVD virtual desktop controller works with OVP together, the built-in domain services provides centralized desktop user authentication and desktop management automation, controls OVP doing operations such as creating, updating, restoring of virtual desktop, and so on. Virtual desktop is delivered safely, fast, reliably to thin client not dependent on the virtual machine network.

Cloud terminal thin client: whether it is a small size, low power of thin client, or a PC, laptop, intelligent terminal,they all can be a software carrier for the cloud terminal thin client , and can be connected to the virtual desktop anytime and anywhere for office applications.

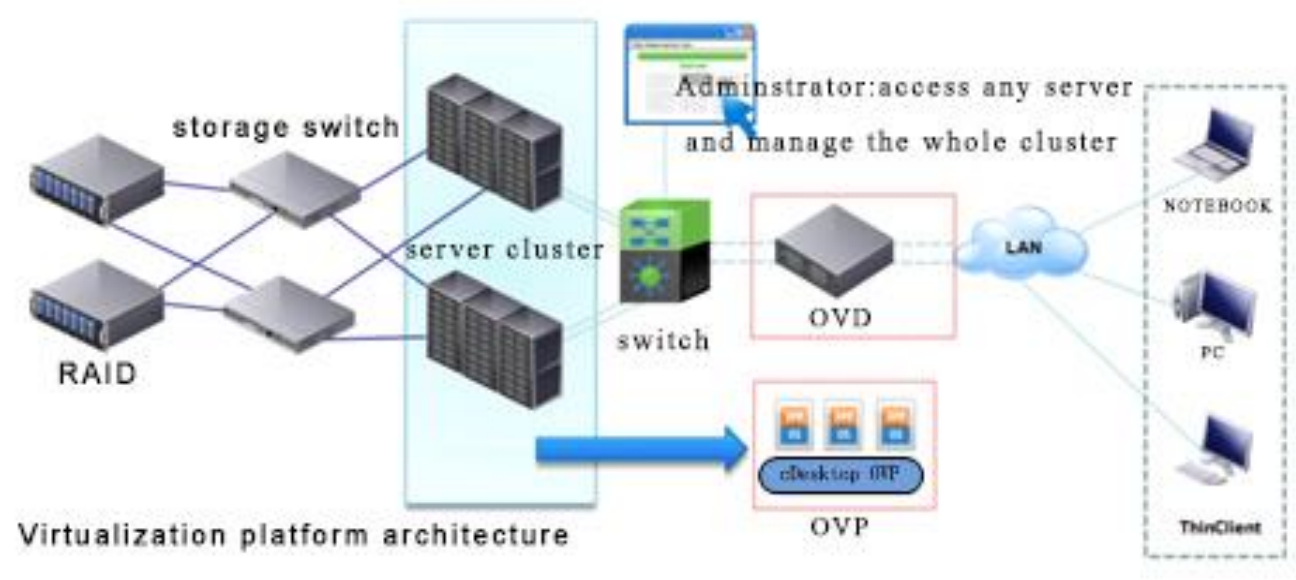

Figure 1. The Overall Architecture Diagram of Cloud Platform

\section{Intelligent Cloud Platform Management}

\subsection{Design of the Cloud Terminal}

From the software design of the cloud terminal, it is called as intelligent cloud terminal. As in addition to the hardware interface of traditional PC host, also originally joined the user experience status indicators. It is occurred a variety of hardware or network problems in the use of cloud desktop, an intelligent alarm can help users understand the operation situation of cloud desktop, when a fault occurs, it can easily be debugged, and clearly feedback failure to administrator, help administrators to quickly 
locate problems and restore business in time, the specific hardware parameters is in the following table.

Table 1. Hardware Parameters of Cloud Terminal

\begin{tabular}{|c|c|c|c|}
\hline Name of Light & Green (flash) & Green（on） & Light Off \\
\hline Power Light & No & Normal & No electricity \\
\hline Terminal Status Light & $\begin{array}{l}\text { External device } \\
\text { connection not normal }\end{array}$ & System start normal & $\begin{array}{l}\text { System could not start } \\
\text { properly }\end{array}$ \\
\hline Wifi Light & Wifi can not connect & Wifi normal & Wifi disable \\
\hline OVD Connection Light & OVD can not connect & $\begin{array}{l}\text { OVD connect } \\
\text { properly }\end{array}$ & $\begin{array}{l}\text { Local network is not } \\
\text { normal }\end{array}$ \\
\hline $\begin{array}{l}\text { Virtual Machine } \\
\text { Experience Status Light }\end{array}$ & $\begin{array}{l}\text { Virtual machine } \\
\text { experience bad } \\
\text { (maybe :network speed } \\
\text { is slow) }\end{array}$ & $\begin{array}{l}\text { Virtual machine } \\
\text { normal }\end{array}$ & $\begin{array}{l}\text { Virtual machine can not } \\
\text { connect }\end{array}$ \\
\hline
\end{tabular}

According to the different states of the indicator light such as flashing, dark, bright, it is able to distinguish different situations conditions affecting the quality of service. According to the status of light, users can easily determine the cause of the failure.

\subsection{Cloud Desktop Deployment}

OVP, OVD are on the same server, thus doing operation on one server can complete the server virtualization and desktop virtualization deployment platform, creating, updating and restoring of the all desktop are completed on the OVD. Compared with the traditional platform virtualization vendors, deployment time is reduced more than $50 \%$ of the installation steps.

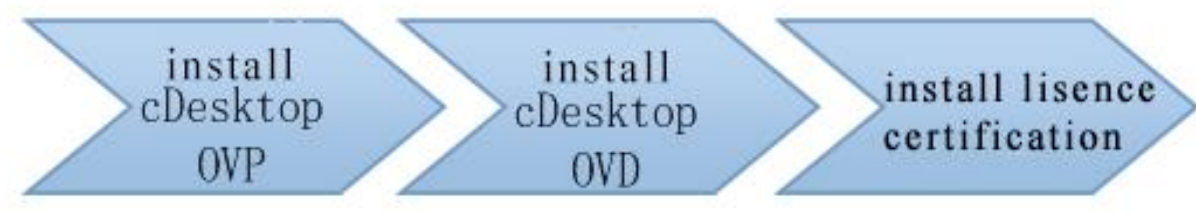

Figure 2. Cloud Desktop Deployment Diagram

At the same time, the built-in local server in the platform easily realizes the user authorization and management without installing the $\mathrm{AD}$ domain.

\subsection{Cloud Terminal Management}

In addition to the unified deployment and management of virtual desktop, use "cloud terminal + cloud desktop" deployment mode, CDesktop OVM is of a cloud terminal management system for enterprise users, it provides easy-to-use for IT management personnel organization and management, control, and the cloud terminal equipment to update the OS image, software distribution, and running state monitoring, can support tens of thousands of orders of magnitude terminal equipment running thin client software, whether it is a thin client, or PC which runs thin client software. 
Table 2. The Statistics Table of Cloud Terminal Management

\begin{tabular}{|c|c|c|c|}
\hline Intelligent Management & Enhance Safety Design & $\begin{array}{l}\text { Accurate Condition } \\
\text { Monitoring }\end{array}$ & Efficient Eextension \\
\hline $\begin{array}{l}\text { Automatic discovery of } \\
\text { terminal equipment; } \\
\text { Automatic update and } \\
\text { upgrade of the equipment; } \\
\text { Adaptive management of } \\
\text { terminal Settings; } \\
\text { Remote boot, shut down } \\
\text { and restart of terminal } \\
\text { equipment; }\end{array}$ & $\begin{array}{l}\text { Communication } \\
\text { encryption, image } \\
\text { updates HTTPS based; } \\
\text { Group management of } \\
\text { system permissions,OVD } \\
\text { linkage,Avoid } \\
\text { duplication } \\
\text { configuration } \\
\text { Device to perform } \\
\text { functions authorized } \\
\text { management; } \\
\text { unified management of } \\
\text { password; }\end{array}$ & $\begin{array}{l}\text { Fine-grained assets } \\
\text { report; } \\
\text { The real-time status } \\
\text { monitoring of } \\
\text { equipment operation; } \\
\text { Comprehensive } \\
\text { operation log audit; }\end{array}$ & $\begin{array}{l}\text { Support } 5000 \text { sets of } \\
\text { cloud terminal } \\
\text { equipment, up to } \\
100000 ; \\
\text { Configuration } \\
\text { management } \\
\text { distribution based on } \\
\text { group,Achieve rapid } \\
\text { deployment } \\
\text { large-scale terminal } \\
\text { equipment and update; } \\
\text { Realize the rapid push } \\
\text { and execution of the } \\
\text { configuration file based } \\
\text { on MAC address, or } \\
\text { other distribution rules; }\end{array}$ \\
\hline
\end{tabular}

\subsection{Cross-regional Centralized Management}

For branch staff are scattered around the country, and the number of the staff in each branch is small, generally do not have IT administrators, and it is difficult for the administrator in headquarters to control the desktop system for efficient management and maintenance at this time. Therefore branch using distributed deployment and centralized management of the thin client and virtual desktop access, it is efficient and without too much intervention of the administrator, and the follow-up operations are finished in the data center branch, new staff can be directly assigned with thin client and the administrator can realize the deployment of the desktop environment within 10 minutes. 


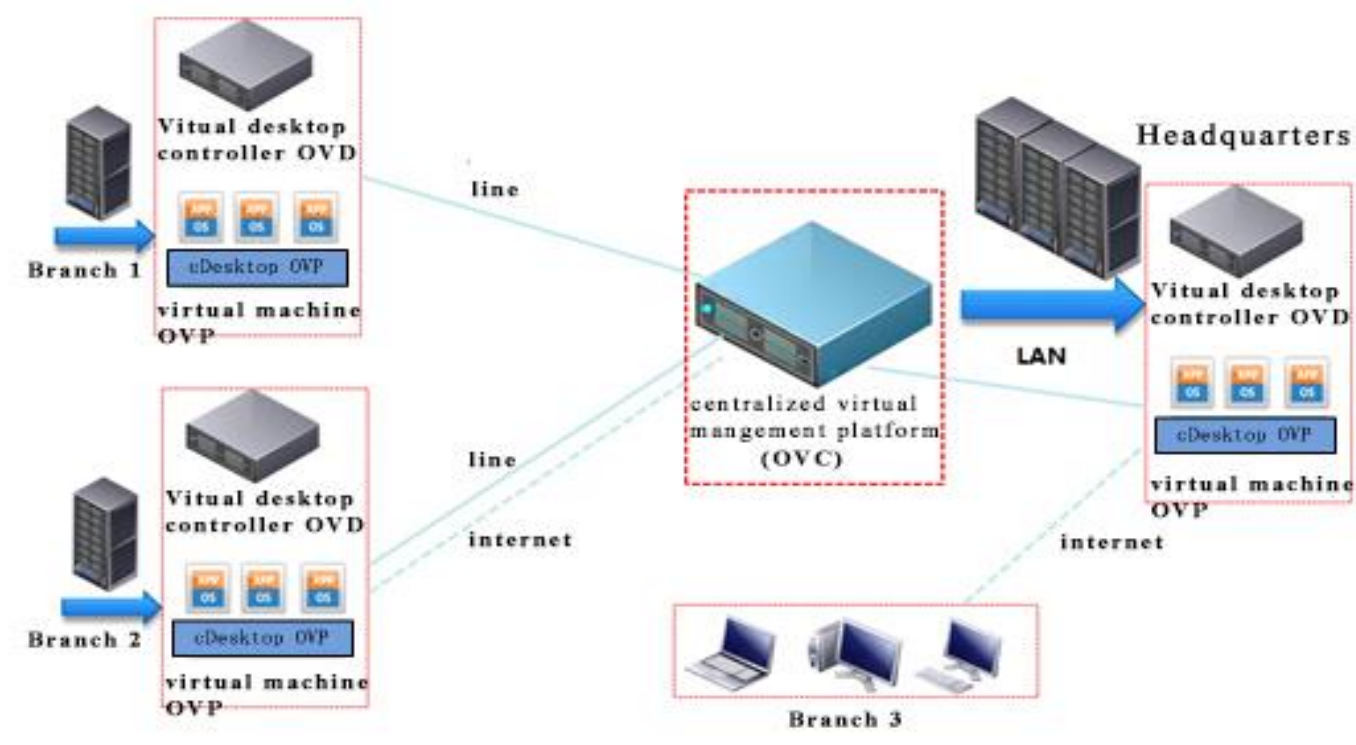

Figure 3. Management Structure of the Cross-regional Cloud Platform

The branch are interconnected with the lines of WAN, bandwidth resources are limited, it can avoid transmission of non-business data in the wan by distributed deployment and centralized management of the desktop cloud, it can occupy less Internet bandwidth and ensure user experience in the branch at the same time. The small branches and scattered personnel can use the VPN access to the virtual desktop platform of the headquarters, through the efficient transport protocol VDX, can guarantee properly access the virtual desktop and application in the condition of narrow bandwidth, so as to achieve the uniform access.

\section{The Safety Design of the Cloud to the End}

\subsection{Platform Security}

Tell from the whole authentication architecture, the third party certification of thin client, OVP and OVD and through SSL encryption communication is embedded into the VDX protocol; it ensures the safety of the whole platform and prevents man-in-the-middle attack (MITM). Even if the user in the process of landing is occurred a middleman interception, because it cannot complete the certification with the OVP, OVD at the same time, will eventually show $\log$ in failure and is unable to enter the virtualization platform.

From the safety of the virtual machine within the cloud desktop, per user exclusively occupies in a virtual machine, realize CPU scheduling, memory, network access, disk IO, the storage space isolation through the OVP mechanisms, the virtual machine failure and safety issues of the users will not affect the others, ensure the safety of isolation between virtual machine; Every user can be assigned personal data disk to hold the documents, when the user migrates to the use of the virtual desktop mode, all data is stored in the data center. Therefore, the personal data is encrypted, and then other users including administrators cannot access the private data and can ensure the safety of users' personal privacy. 
From the safety perspective of the management of the virtual machine, different administrator role are granted different privileges, and save the operation log. Support hierarchical management authority, including the superior administrator will have the right to operate the subordinate administrators' behavior and not vice versa; the superior administrator have right grant resources authorization of the virtual desktop to the subordinate administrator [2].

\subsection{Transmission Security}

The cloud desktop image information not user data is transferred from the server to the terminal network, user data is not real, the image information has been SSL encrypted in the process the network transmission, and usually can't be cracked easily. At the same time, as the image is transferred, so even if it is intercepted, still the original user data can not be gotten, and ensure the safety of data to the greatest extent.

\subsection{Terminal Security}

It can be parsed from two aspects of hardware and software for the cloud terminal security, and the concrete structure is as shown in figure 4.

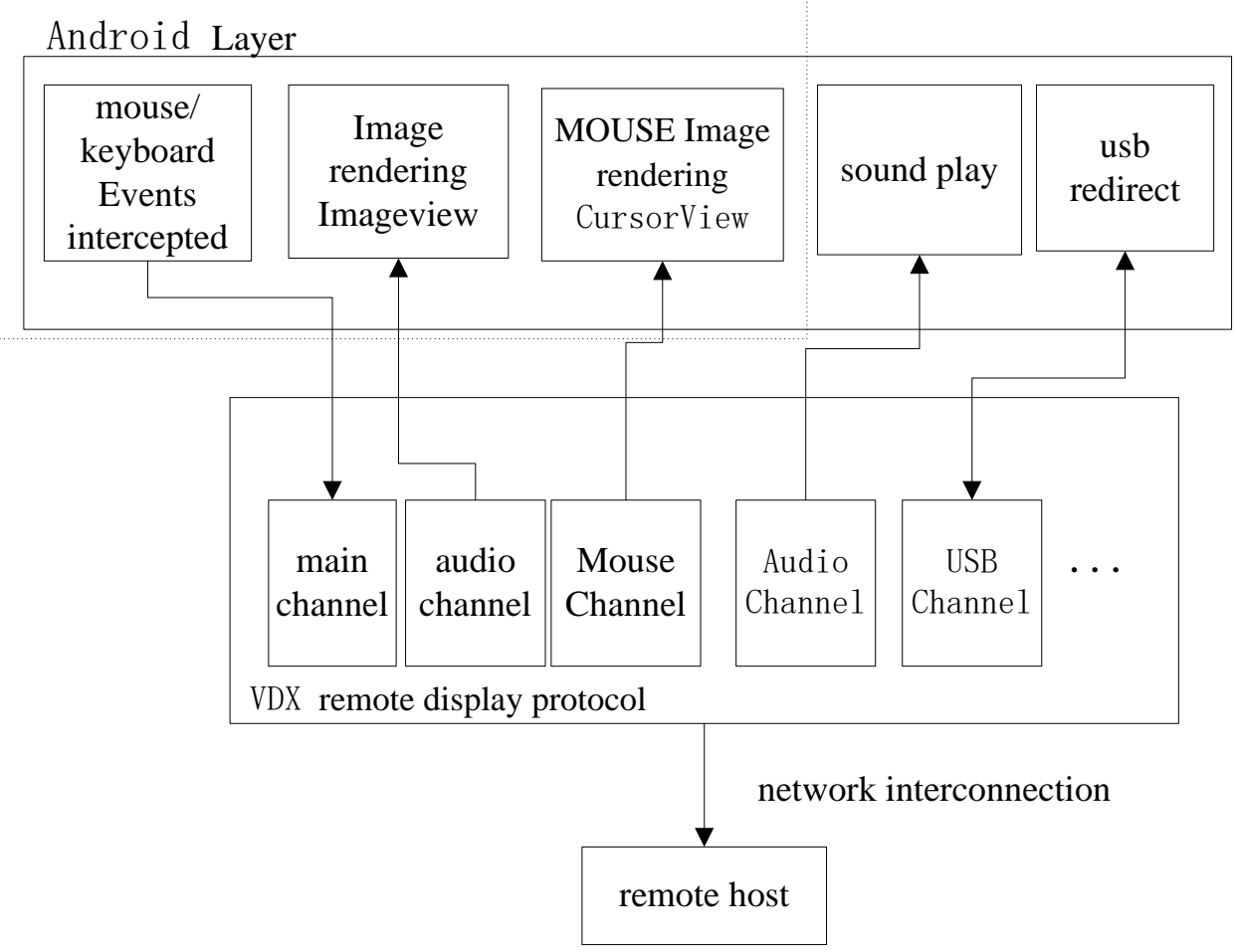

Figure 4. Cloud Terminal Data Channels

From the hardware perspective as the cloud terminal itself does not make any calculations, only makes image coding and decoding operations, does not have the hard disk and data is not stored in the cloud terminal, thus fundamentally eliminates the data leak problem.

From the software perspective, through the USB access control, it is ensured that data in virtual desktop is set by the administrator permissions to ban, and to allow the corresponding USB copy. At the same time, operating system of the cloud terminal is the reinforcement lightweight Linux operating system, a closed system is less vulnerabilities and the system is intruded by hacker and Trojans much less than the Windows operating system, so as to guarantee the safety of terminal system. 


\section{The Stability Design of Point to Surface}

The management node of the traditional desktop virtualization platform must be a single server or virtual machine, once the malfunction of the management platform occurred, it will lose control of the whole virtualization platform, there is a serious risk of single point of failure.

In CDesktop OVD desktop cloud platform, while using cluster pattern deployment server, each server in the cluster is equal, there is no central management node, each node will be synchronized with each other by the multicast information, log in to any one node is able to do management for the whole cluster. So as to realize the high availability of the platform.

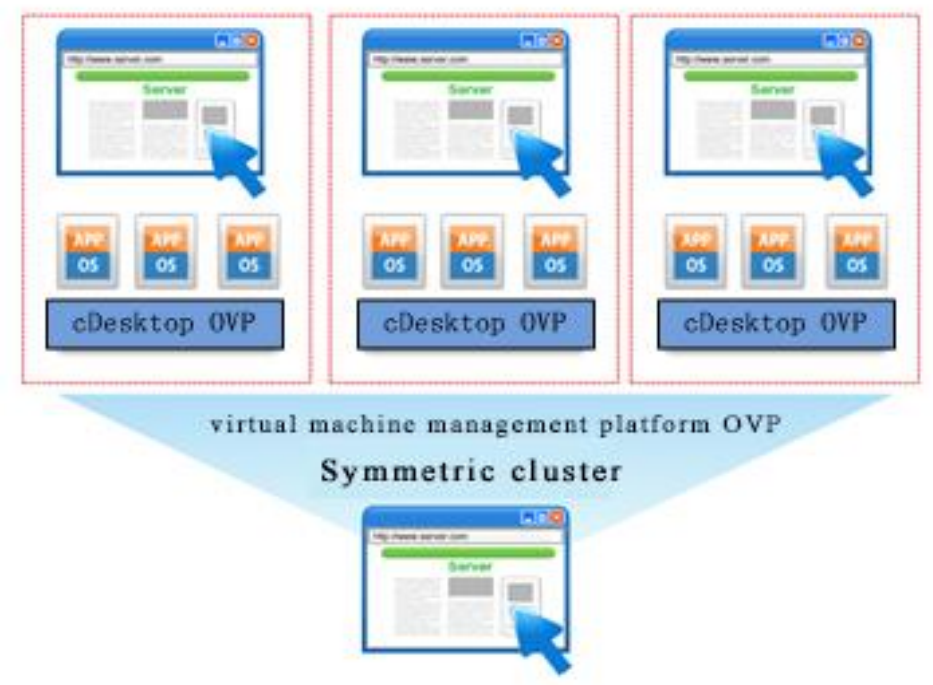

Figure 5. Cluster Deployment

The state information and configuration files are synchronized within the cluster, thus there is not large flow of data, it is not occured network congestion because of the synchronization of information .

\section{Highlights Analysis of Virtual Experiment Platform}

Using the development of modern cloud technology, the virtualization of the cloud platform system laboratory has more unique advantages than traditional computer lab, and also has the characteristics of conventional computer (PC) labs, the following three representative highlights are respectively listed as follows:

the hd video experience

In CDesktop OVD desktop cloud platform, the image not the real application data is transferred from the server to terminal transmission, so efficient transmission of image with the limited bandwidth determines the user experience when user are using cloud desktop. In virtualization server it is used a unique multimedia heuristic recognition algorithm, can distingwish the the GIF animation ads, video, window interface change speed, etc., intelligently identify real video content, use special video compression coding, and are sent to the client, the rest is transferred as the picture frame. CDesktop OVD support $1080 \mathrm{p}$ hd video on the local broadcast and online play inside the virtual desktop, 
the actual effect is exactly the same as that of the PC, so that users can not feel it is in the use of cloud desktop, can satisfy the user's daily use of various application environments [3] [4][5].

efficient VDX protocal

VDX (Virtual Desktop eXtend, Virtual Desktop extension protocol) is an optimum connection protocol that is VDI architechture oriented customized Desktop access, designed to overcome the existing faluts of desktop access protocol (RDP/ICA/PCoIP/SPICE etc.) in a virtual desktop architecture environment that it is lack of traditional PC desktop function and the weaknesses of gap of experience including video multimedia fluency and peripherals supporting, etc. The current mainstream desktop access protocol is designed before the birth of VDI structure, without considering the demand of specific scenarios in the process of access of VDI desktop, the VDX protocal is tailored specifically for VDI architecture, solves the key problems such as broadcast of video multimedia and support of various ancillary, and learn from the advantages of the RDP and SPICE open protocols, are integrated into a more suitable protocal for VDI deployment, provide better bandwidth, better user experience, more similar to traditional PC peripherals [7] [8] .

consistent with pc usage

It is occurred the problem that using habit is not consistant during the switch from PC mode to cloud desktop mode, CDesktop OVD did a lot of experience improvement work. There are the following points: first, allowed to change the password of the login user; Second, the user can power off/stop/start their own virtual machine; Third, the network cloud desktop does not depend on network itself, can disable or change the network configuration; Fourth, the cloud desktop system boot screen can be seen; Fifth, cloud desktop is automatically connected after it boots[9][10].

\section{Conclusion}

Cloud laboratory is the trend, no matter the hardware and software cost or the late computer maintenance, management and upgrade, the cloud computer laboratory is better than the traditional physical machine labs. The cloud lab has a advantage in many aspects, especially in data management, distributed data processing, remote management and maintenance of computer and so on. In the next few years, with the rapid development of cloud computing technology, we will continue to have new technological change in the virtualization technology as the basis of the cloud computing infrastructure, and gradually enhance openness, security, compatibility, the virtual laboratory based on clound platform will have a better development, making the virtual lab appeared in a variety of areas, especially in the medium and large enterprises and universities.

\section{Acknowledgement}

Fund Project: The National Natural Science Foundation of China (61472166), Changzhou Cloud Computing and Intelligent Information Processing Laboratory funded projects (CM20123004). 


\section{References}

[1] C. H. Huang and Y. Q. Lin, "Research and implementation of cloud-based virtual computer lab", Research and Exploration of Laboratory, vol. 11, no. 22, (2010).

[2] L. Li, J. W. Liao and L. Ou, "Cloud Computing Discussion", Application Research of Computers, vol. 12, no. 27, (2012).

[3] Q. W. Meng and T. Liu, "The model research of computer laboratory construction based on Cloud Computing", Intelligent Computer Application, vol. 12, no. 36, (2011).

[4] H. J. Liao, Y. B. Yang and L. Z. Tang, "Research and implementation of animation rendering experimental platform based on Cloud Computing”, Rresearch and Exploration of Laboratory, vol. 7, no. 10, (2012).

[5] G. L. Li and Yan Zhang, "Computer laboratory construction based on Cloud Computing”, Journal f Henan Mechanical and Electrical Engineering College, vol. 5, no. 12, (2011).

[6] L. J. Yang and S. P. Yang, "Dynamic Migration Technology Platform in the Cloud Computer Forensics Applied Research", Advanced Technologies, Embedded and Multimedia for Human-centric Computing Lecture Notes in Electrical Engineering, vol. 12, no. 60, (2013).

[7] B. Kerrigan and Y. Chen, "A Study of Entropy Sources in Cloud Computers: Random Number Generation on Cloud Hosts", Computer Network Security, vol. 10, no. 40, (2012).

[8] G. H. Xiong, "Designation of Green Computer Terminal Supported by Cloud Computing Testbeds and Research Infrastructure”, Development of Networks and Communities, vol. 10, no. 40, (2014).

[9] N. Sabharwal and P. Wali, "Cloud Capacity Management", Cloud Capacity Management, vol. 7, no. 14, (2013).

[10] Z. Q. Dong, N. Liu and R. R. Cessa, "Greedy scheduling of tasks with time constraints for energy-efficient cloud-computing data centers", Journal of Cloud Computing, vol. 3, no. 7, (2015).

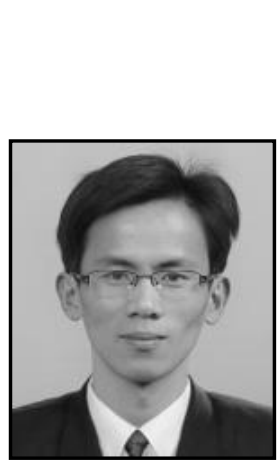

\section{Authors}

\section{Wang Ke Feng}

Author introduction: Wang Kefeng (1981-), male, Jiangsu Changzhou, lecturer, master. The main research direction: Graphic Encryption,Information Security.

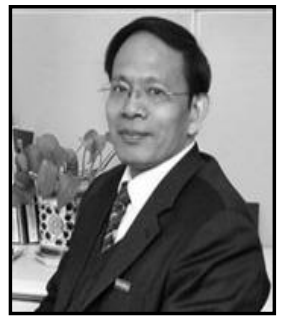

Ye FeiYue

Author introduction: Ye FeiYue (1968-), male, Jiangsu Changzhou, professor, doctor. The main research direction: Big data,Graphic Encryption,Information Security. 
International Journal of Smart Home

Vol. 9, No. 11, (2015) 